\title{
Herschel/HIFI discovery of interstellar chloronium $\left(\mathrm{H}_{2} \mathrm{Cl}^{+}\right)^{\star, \star \star}$
}

D. C. Lis ${ }^{1}$, J. C. Pearson ${ }^{13}$, D. A. Neufeld ${ }^{3}$, P. Schilke ${ }^{8,12}$, H. S. P. Müller ${ }^{12}$, H. Gupta ${ }^{13}$, T. A. Bell ${ }^{1}$, C. Comito ${ }^{8}$, T. G. Phillips ${ }^{1}$, E. A. Bergin ${ }^{2}$, C. Ceccarelli ${ }^{6}$, P. F. Goldsmith ${ }^{13}$, G. A. Blake ${ }^{1}$, A. Bacmann ${ }^{6,23}$, A. Baudry ${ }^{23}$, M. Benedettini ${ }^{24}$, A. Benz ${ }^{37}$, J. Black ${ }^{36}$, A. Boogert ${ }^{16}$, S. Bottinelli ${ }^{4,5}$, S. Cabrit ${ }^{25}$, P. Caselli ${ }^{26}$, A. Castets ${ }^{6}$, E. Caux ${ }^{4,5}$, J. Cernicharo ${ }^{7}$, C. Codella ${ }^{27}$, A. Coutens ${ }^{4,5}$, N. Crimier ${ }^{6,7}$, N. R. Crockett ${ }^{2}$, F. Daniel ${ }^{7,9}$, K. Demyk ${ }^{4,5}$, C. Dominic ${ }^{28,29}$, M.-L. Dubernet ${ }^{10,11}$, M. Emprechtinger ${ }^{1}$, P. Encrenaz ${ }^{25}$, E. Falgarone ${ }^{9}$, A. Fuente ${ }^{30}$, M. Gerin ${ }^{9}$, T. F. Giesen ${ }^{12}$, J. R. Goicoechea ${ }^{7}$, F. Helmich ${ }^{20}$, P. Hennebelle ${ }^{9}$, Th. Henning ${ }^{45}$, E. Herbst ${ }^{14}$, P. Hily-Blant ${ }^{6}, \AA$ A. Hjalmarson ${ }^{38}$, D. Hollenbach ${ }^{39}$, T. Jack ${ }^{23}$, C. Joblin ${ }^{4,5}$, D. Johnstone ${ }^{15}$, C. Kahane ${ }^{6}$, M. Kama ${ }^{28}$, M. Kaufman ${ }^{40}$, A. Klotz ${ }^{4,5}$, W. D. Langer ${ }^{13}$, B. Larsson ${ }^{41}$, J. Le Bourlot ${ }^{42}$, B. Lefloch ${ }^{6}$, F. Le Petit ${ }^{42}$, D. Li ${ }^{13}$, R. Liseau ${ }^{36}$, S. D. Lord ${ }^{16}$, A. Lorenzani ${ }^{24}$, S. Maret ${ }^{6}$, P. G. Martin ${ }^{17}$, G. J. Melnick ${ }^{18}$, K. M. Menten ${ }^{8}$, P. Morris ${ }^{13}$, J. A. Murphy ${ }^{19}$, Z. Nagy ${ }^{21}$, B. Nisini ${ }^{31}$, V. Ossenkopf ${ }^{12,20}$, S. Pacheco ${ }^{6}$, L. Pagani ${ }^{25}$, B. Parise ${ }^{8}$, M. Pérault ${ }^{9}$, R. Plume ${ }^{21}$, S.-L. Qin ${ }^{12}$, E. Roueff ${ }^{42}$, M. Salez ${ }^{25,44}$, A. Sandqvist ${ }^{43}$, P. Saraceno ${ }^{32}$, S. Schlemmer ${ }^{12}$, K. Schuster ${ }^{33}$, R. Snell ${ }^{22}$, J. Stutzki ${ }^{12}$, A. Tielens ${ }^{34}$, N. Trappe ${ }^{19}$, F. F. S. van der Tak ${ }^{21,46}$, M. H. D. van der Wiel $^{21,46}$, E. van Dishoeck ${ }^{34}$, C. Vastel ${ }^{4,5}$, S. Viti ${ }^{35}$, V. Wakelam ${ }^{23}$, A. Walters ${ }^{4.5}$, S. Wang ${ }^{2}$, F. Wyrowski ${ }^{8}$, H. W. Yorke ${ }^{13}$, S. Yu ${ }^{13}$, J. Zmuidzinas ${ }^{1}$, Y. Delorme ${ }^{44}$, J.-P. Desbat ${ }^{23}$, R. Güsten ${ }^{8}$, J.-M. Krieg ${ }^{44}$, and B. Delforge ${ }^{44}$

(Affiliations are available on page 5 of the online edition)

Received 9 May 2010 / Accepted 14 June 2010

\section{ABSTRACT}

We report the first detection of chloronium, $\mathrm{H}_{2} \mathrm{Cl}^{+}$, in the interstellar medium, using the HIFI instrument aboard the Herschel Space Observatory. The $2_{12}-1_{01}$ lines of ortho- $\mathrm{H}_{2}^{35} \mathrm{Cl}^{+}$and ortho- $\mathrm{H}_{2}^{37} \mathrm{Cl}^{+}$are detected in absorption towards NGC $6334 \mathrm{I}$, and the $1_{11}-0_{00}$ transition of para- $\mathrm{H}_{2}^{35} \mathrm{Cl}^{+}$is detected in absorption towards NGC 6334I and Sgr B2(S). The $\mathrm{H}_{2} \mathrm{Cl}^{+}$column densities are compared to those of the chemically-related species $\mathrm{HCl}$. The derived $\mathrm{HCl} / \mathrm{H}_{2} \mathrm{Cl}^{+}$column density ratios, $\sim 1-10$, are within the range predicted by models of diffuse and dense photon dominated regions (PDRs). However, the observed $\mathrm{H}_{2} \mathrm{Cl}^{+}$column densities, in excess of $10^{13} \mathrm{~cm}^{-2}$, are significantly higher than the model predictions. Our observations demonstrate the outstanding spectroscopic capabilities of HIFI for detecting new interstellar molecules and providing key constraints for astrochemical models.

Key words. astrochemistry - line: identification - ISM: abundances - ISM: molecules - molecular processes - submillimetre: ISM

\section{Introduction}

The halogen elements, fluorine and chlorine, form hydrides that are very strongly bound: hydrogen fluoride is the only diatomic hydride, and $\mathrm{HCl}^{+}$the only diatomic hydride cation, with a dissociation energy exceeding that of molecular hydrogen. Drawing upon earlier work by Jura (1974), Dalgarno et al. (1974), van Dishoeck \& Black (1986), Blake et al. (1986), Schilke et al. (1995), Federman et al. (1995), and Amin (1996), Neufeld \& Wolfire (2009; hereafter NW09) have recently carried out a theoretical study of the chemistry of chlorine-bearing molecules, in both diffuse and dense molecular clouds. In diffuse interstellar gas clouds, the dominant ionization state of every element is determined by its ionization potential. Chlorine, with an ionization potential slightly lower than that of hydrogen, is predominantly singly-ionized. The $\mathrm{Cl}^{+}$ion can react exothermically

* Herschel is an ESA space observatory with science instruments provided by European-led Principal Investigator consortia and with important participation from NASA.

$\star \star$ Table 1 and acknowledgments (page 5) are only available in electronic form at http://www. aanda.org with $\mathrm{H}_{2}$, the dominant molecular constituent of the interstellar medium (ISM):

$\mathrm{Cl}^{+}+\mathrm{H}_{2} \rightarrow \mathrm{HCl}^{+}+\mathrm{H}$.

The product of this reaction is the reactive $\mathrm{HCl}^{+}$ion, which undergoes further reaction with $\mathrm{H}_{2}$ to form $\mathrm{H}_{2} \mathrm{Cl}^{+}$:

$\mathrm{HCl}^{+}+\mathrm{H}_{2} \rightarrow \mathrm{H}_{2} \mathrm{Cl}^{+}+\mathrm{H}$.

The $\mathrm{H}_{2} \mathrm{Cl}^{+}$molecule does not react with $\mathrm{H}_{2}$, and is destroyed by dissociative recombination and proton transfer to $\mathrm{CO}$, both of which are sources of hydrogen chloride, $\mathrm{HCl}$.

Prior to the launch of Herschel, the $\mathrm{H}^{35} \mathrm{Cl}$ and $\mathrm{H}^{37} \mathrm{Cl}$ isotopologues were the only chlorine-containing molecules to have been detected in the ISM (e.g., Blake et al. 1985; Zmuidzinas et al. 1995; Schilke et al. 1995; Salez et al. 1996; see also recent HIFI observations of Cernicharo et al. 2010) ${ }^{1}$. However, predictions for the chemistry of Cl-bearing interstellar molecules

\footnotetext{
1 The metal halides $\mathrm{NaCl}, \mathrm{KCl}$, and $\mathrm{AlCl}$ have been detected in the circumstellar envelope of the evolved star IRC +10216 , with abundances that reflect the thermochemical equilibrium established within the stellar photosphere (Cernicharo \& Guélin 1987).
} 
(NW09) have identified chloronium, $\mathrm{H}_{2} \mathrm{Cl}^{+}$, as a relatively abundant species that is potentially detectable. $\mathrm{H}_{2} \mathrm{Cl}^{+}$is predicted to be most abundant in those environments where the ultraviolet radiation is strong: in diffuse clouds, or near the surfaces of dense clouds that are illuminated by nearby $\mathrm{O}$ and $\mathrm{B}$ stars. In such environments, the photoionization of atomic chlorine leads to a large abundance of $\mathrm{Cl}^{+}$ions that can form $\mathrm{HCl}^{+}$and $\mathrm{H}_{2} \mathrm{Cl}^{+}$ through reactions (1) and (2). A secondary abundance peak occurs in dense, shielded regions; here $\mathrm{HCl}$ becomes a significant reservoir of gas-phase chlorine, and can produce $\mathrm{H}_{2} \mathrm{Cl}^{+}$through reaction with $\mathrm{H}_{3}^{+}$:

$\mathrm{HCl}+\mathrm{H}_{3}^{+} \rightarrow \mathrm{H}_{2} \mathrm{Cl}^{+}+\mathrm{H}_{2}$.

However, the chlorine depletion is typically large within such regions (Schilke et al. 1995) and thus the overall $\mathrm{H}_{2} \mathrm{Cl}^{+}$abundance is rather small.

In diffuse molecular clouds of density $n_{\mathrm{H}}=10^{2.5} \mathrm{~cm}^{-3}, \mathrm{H}_{2}$ column density $\geq 10^{20} \mathrm{~cm}^{-2}$, and $\chi_{\mathrm{UV}}$ in the range $1-10$ (where $\chi_{\mathrm{UV}}$ is the UV radiation field normalized with respect to the mean interstellar value, Draine 1978), the NW09 model predicts $\mathrm{H}_{2} \mathrm{Cl}^{+}$column densities $\sim 3 \times 10^{10} \chi_{\mathrm{UV}} \mathrm{cm}^{-2}$. In dense PDRs $\left(n_{\mathrm{H}}=10^{4} \mathrm{~cm}^{-3}\right)$ illuminated by strong radiation fields $\left(\chi_{\mathrm{UV}}>\right.$ $10^{3}$ ), the predicted $\mathrm{H}_{2} \mathrm{Cl}^{+}$column densities are $\sim 10^{12} \mathrm{~cm}^{-2}$.

In this Letter, we report the first detection of chloronium towards NGC 6334I and Sgr B2(S), obtained using the HIFI instrument (de Graauw et al. 2010) aboard the Herschel Space Observatory (Pilbratt et al. 2010). NGC 6334 is a luminous and relatively nearby $(1.7 \mathrm{kpc})$ molecular cloud/H II region complex containing several concentrations of massive stars at various stages of evolution. The far-infrared source "I", located at the northeastern end of the complex, is associated with a NIR cluster of bolometric luminosity of $2.6 \times 10^{5} L_{\odot}$ (Sandell 2000), with four embedded compact millimeter continuum sources (Hunter et al. 2006). Sgr B2(S) is a strong submillimeter continuum source with a much less complex hot core emission spectrum, as compared to its better known neighbor Sgr B2(M). This makes it a prime candidate for absorption studies, probing the entire sight-line between the Sun and the Galactic center, with clouds in the Orion, Sagittarius, and Scutum spiral arms easily identified at separate velocities (e.g., Greaves \& Nyman 1996).

\section{Observations}

HIFI observations presented here were carried out between 2010 March 1 and March 23, using the dual beam switch (DBS) observing mode, as part of guaranteed and open time key programs CHESS: Chemical HErschel Spectral Surveys, HEXOS: Herschel/HIFI observations of EXtra-Ordinary Sources: The Orion and Sagittarius B2 starforming regions, and HOP: Herschel oxygen program. The source coordinates are: $\alpha_{J 2000}=$ $17^{\mathrm{h}} 20^{\mathrm{m}} 53.32^{\mathrm{s}}$ and $\delta_{J 2000}=-35^{\circ} 46^{\prime} 58.5^{\prime \prime}$ for NGC 6334I, and $\alpha_{J 2000}=17^{\mathrm{h}} 47^{\mathrm{m}} 20.3^{\mathrm{s}}$ and $\delta_{J 2000}=-28^{\circ} 23^{\prime} 43.0^{\prime \prime}$ for Sgr B2(S). The DBS reference beams lie approximately $3^{\prime}$ east and west (i.e. perpendicular to the roughly north-south elongation of the two sources). Because the DBS mode alternates between two reference positions, separated by $6^{\prime}$ on the sky, we used the Level 1 data to compute a difference spectrum between the two reference positions to check for possible contamination in the reference beams; we see no evidence for emission or absorption in such a difference spectrum. We used the HIFI wide band spectrometer (WBS) providing a spectral resolution of $1.1 \mathrm{MHz}$ $\left(\sim 0.4 \mathrm{~km} \mathrm{~s}^{-1}\right.$ at $\left.780 \mathrm{GHz}\right)$ over a $4 \mathrm{GHz}$ IF bandwidth. The spectra presented here are averages of the $\mathrm{H}$ and $\mathrm{V}$ polarizations, with equal weighting, reduced using HIPE (Ott 2010) with pipeline version 2.6. The resulting Level 2 DSB spectra were exported to the FITS format for a subsequent data reduction and analysis using the IRAM GILDAS package (http://iram. fr/IRAMFR/ GILDAS).

The band $2 \mathrm{~b}, 1 \mathrm{~b}$ and $1 \mathrm{a}$ spectral scans of NGC 6334I consist of double sideband spectra (DSB) with a redundancy of 8 , which gives observations of a lower or upper sideband frequency with 8 different settings of the local oscillator (LO). The Sgr B2(S) data consist of $8 \mathrm{LO}$ settings with a high redundancy of 12, centered near the frequency of the $487.2 \mathrm{GHz}$ line of $\mathrm{O}_{2}$. The observations were fine-tuned so that 4 of the $8 \mathrm{LO}$ settings cover the frequency of the $\mathrm{p}-\mathrm{H}_{2} \mathrm{Cl}^{+}$line. These observing modes allow for the deconvolution and isolation of a single sideband spectrum (Comito \& Schilke 2002). We applied the standard deconvolution routine within CLASS. All NGC 6334I data presented here are deconvolved single sideband spectra, including the continuum. The $\mathrm{HCl}$ data in Sgr B2(S) were obtained using the DBS single point observing mode with 3 shifted LO settings that were averaged to produce the final spectrum. The HIFI beam size at $485 \mathrm{GHz}$ and $780 \mathrm{GHz}$ is $44^{\prime \prime}$ and $30^{\prime \prime}$, respectively, with main beam efficiency of $\sim 0.68$.

\section{Spectroscopy of $\mathrm{H}_{2} \mathrm{Cl}^{+}$}

The $\mathrm{H}_{2} \mathrm{Cl}^{+}$ion is a closed-shell molecule, isoelectronic with $\mathrm{H}_{2} \mathrm{~S}$. Like $\mathrm{H}_{2} \mathrm{~S}$ and water, $\mathrm{H}_{2} \mathrm{Cl}^{+}$is a highly asymmetric top, exhibiting a b-type rotational spectrum. Its fairly large dipole moment, calculated ab initio to be 1.89 D (Müller et al. 2005), about $70 \%$ larger than that of $\mathrm{HCl}(1.109 \mathrm{D}$; de Leluw \& Dymanus 1973), results in strong lines in the THz range. Araki et al. (2001) measured rotational spectra of $\mathrm{H}_{2}^{35} \mathrm{Cl}^{+}, \mathrm{H}_{2}{ }^{37} \mathrm{Cl}^{+}$, and $\mathrm{HDCl}^{+}$ below $500 \mathrm{GHz}$. The accurate spectroscopic constants derived from these measurements, including electric quadrupole coupling parameters, yield a central bond angle in $\mathrm{H}_{2} \mathrm{Cl}^{+}$of $\sim 94.2^{\circ}$ (similar to that of $\mathrm{H}_{2} \mathrm{~S}, 92.2^{\circ}$; Burrus \& Gordy 1953), and permit the prediction of the ground state ortho transitions of $\mathrm{H}_{2} \mathrm{Cl}^{+}$and $\mathrm{H}_{2}{ }^{37} \mathrm{Cl}^{+}$near $780 \mathrm{GHz}$ to well within $1 \mathrm{MHz}$ (see Table 1).

\section{Results}

\subsection{NGC 6334I}

Strong absorption at the frequency of the $2{ }_{12}-1_{01}$ transition of $\mathrm{o}_{-} \mathrm{H}_{2}^{35} \mathrm{Cl}^{+}$at $781.6 \mathrm{GHz}$ in NGC 6334I (Fig. 1a) has provided the initial identification. Fitting the $\mathrm{o}-\mathrm{H}_{2}^{35} \mathrm{Cl}^{+} 2_{12}-1_{01}$ hyperfine structure (HFS) gives a line velocity of $-1.7 \mathrm{~km} \mathrm{~s}^{-1}$ for the strongest hyperfine component and a line width of $11.6 \mathrm{~km} \mathrm{~s}^{-1}$. The $\mathrm{OH}$ absorption profiles (Brooks \& Whiteoak 2001) reveal two molecular clouds located along the line of sight to NGC 6334, one with velocities extending from -15 to $2 \mathrm{~km} \mathrm{~s}^{-1}$, and one with a well-defined velocity near $6 \mathrm{~km} \mathrm{~s}^{-1}$. The hot core emission lines peak at about $-6.5 \mathrm{~km} \mathrm{~s}^{-1}$ (e.g., $\mathrm{C}^{18} \mathrm{O} 7-6$ in the same band; $\mathrm{HCl}$, Sect. 4.1). Water and $\mathrm{CH}$ spectra towards NGC 6334I show multiple velocity components, including absorption near $0 \mathrm{~km} \mathrm{~s}^{-1}$, close to the $\mathrm{H}_{2}^{35} \mathrm{Cl}^{+}$velocity (Emprechtinger et al. 2010; van der Wiel et al. 2010). The chloronium line velocity in NGC 6334I is in good agreement with the $\mathrm{H}_{2} \mathrm{O}^{+}$absorption velocity, also a tracer of diffuse gas, when $\mathrm{H}_{2} \mathrm{O}^{+}$frequencies of Mürtz et al. (1998) are used (see Schilke et al. 2010 for a discussion of the $\mathrm{H}_{2} \mathrm{O}^{+}$line frequencies). The large $\mathrm{H}_{2} \mathrm{Cl}^{+}$line width may be due to blending of multiple absorption components. However, the $\mathrm{H}_{2} \mathrm{O}^{+}$line width is also quite large, about $8 \mathrm{~km} \mathrm{~s}^{-1}$.

The corresponding line of $\mathrm{o}-\mathrm{H}_{2}^{37} \mathrm{Cl}^{+}$is also detected (Fig. 1b). However, the spectrum is contaminated by interfering emission of dimethyl ether (light-blue line in Fig. 1b), one of 


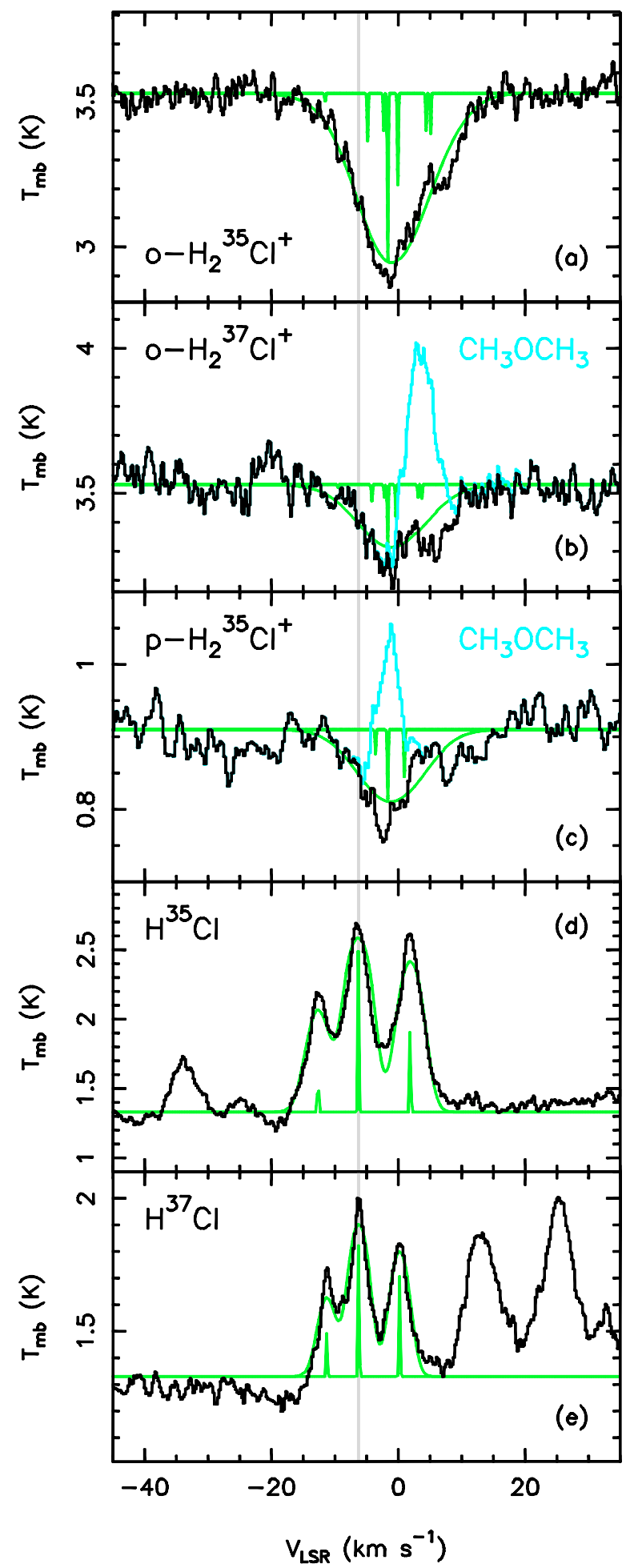

Fig. 1. Spectra of chlorine species in NGC 6334I: a) o- $\mathrm{H}_{2}^{35} \mathrm{Cl}^{+} 2_{12}-1_{01}$; b) $0-\mathrm{H}_{2}^{37} \mathrm{Cl}^{+} 2_{12}-1_{01}$, c) $\mathrm{p}-\mathrm{H}_{2}^{35} \mathrm{Cl}^{+} 1_{11}-0_{00}$, d) $\mathrm{H}^{35} \mathrm{Cl} 1-0$; and e) $\mathrm{H}^{37} \mathrm{Cl}$ $1-0$. The velocity scale corresponds to the strongest HFS components. Green lines show HFS fits and positions of the HFS components. The $\mathrm{o}-\mathrm{H}_{2}^{37} \mathrm{Cl}^{+}$and $\mathrm{p}-\mathrm{H}_{2}^{35} \mathrm{Cl}^{+}$lines are blended with dimethyl ether emission (light-blue lines in panels b) and c).

the most abundant "weeds" in NGC 6334I. The contamination is subtracted by using an LTE model that fits profiles of nearby dimethyl ether lines with similar upper level energies (Endres et al. 2009). The resulting $0-\mathrm{H}_{2}^{37} \mathrm{Cl}^{+}$spectrum is shown as a black line in Fig. 1b. The $1_{11}-0_{00}$ line of $\mathrm{p}-\mathrm{H}_{2}^{35} \mathrm{Cl}^{+}$(Fig. 1c) is also blended with dimethyl ether emission, similarly subtracted.
$\mathrm{H}_{2} \mathrm{Cl}^{+}$spectra in NGC 6334I can be compared to those of the chemically related species $\mathrm{HCl}$ (Fig. 1d and 1e). The lines of $\mathrm{H}^{35} \mathrm{Cl}$ and $\mathrm{H}^{37} \mathrm{Cl}$ are detected in emission at the hot core velocity $\left(\sim-6.3 \mathrm{~km} \mathrm{~s}^{-1}\right)$, with narrow line widths of 4.1 and $3.3 \mathrm{~km} \mathrm{~s}^{-1}$, respectively. The $\mathrm{HCl} \mathrm{HFS}$ is resolved spectrally using HIFI WBS, allowing for the determination of the line opacity.

We have modelled the $\mathrm{H}_{2} \mathrm{Cl}^{+}$spectra assuming the same excitation temperature of $5 \mathrm{~K}$ for all hyperfine components ${ }^{2}$. A low value of the excitation temperature is justified given the high spontaneous emisison rates and critical densities of the transitions considered here and it provides a lower limit for the molecular column densities derived from absorption measurements. An HFS fit to the $2_{12}-1_{01}$ transition of $\mathrm{o}-\mathrm{H}_{2}^{35} \mathrm{Cl}^{+}$(green line in Fig. 1a) gives an $0-\mathrm{H}_{2}^{35} \mathrm{Cl}^{+}$column density of $1.3 \times 10^{13} \mathrm{~cm}^{-2}$, under the assumption that the absorption completely covers the continuum and is not concentrated in small clumps. A fit to the $1_{11}-0_{00}$ spectrum of $\mathrm{p}-\mathrm{H}_{2}^{35} \mathrm{Cl}^{+}$, with all parameters other than the column density fixed, gives a $\mathrm{p}-\mathrm{H}_{2}^{35} \mathrm{Cl}^{+}$column density of $4.0 \times 10^{12} \mathrm{~cm}^{-2}$. The total $\mathrm{H}_{2}^{35} \mathrm{Cl}^{+}$column density is thus $1.7 \times 10^{13} \mathrm{~cm}^{-2}$ and the ortho-to-para ratio is 3.2 , consistent with the statistical weight ratio. For an excitation temperature of $2.7 \mathrm{~K}$, the ortho and para $\mathrm{H}_{2} \mathrm{Cl}^{+}$column densities are approximately $10 \%$ and $20 \%$ lower, respectively. The $\mathrm{H}_{2}^{35} \mathrm{Cl}^{+}$spectra are all optically thin (line center optical depth of $\sim 0.2$ for the ortho line). We derive an $\mathrm{H}_{2}^{35} \mathrm{Cl}^{+} / \mathrm{H}_{2}^{37} \mathrm{Cl}^{+}$ratio of 3 , close to the terrestrial ratio of 3.1.

We have modelled the $\mathrm{H}^{35} \mathrm{Cl}$ and $\mathrm{H}^{37} \mathrm{Cl}$ emission spectra assuming a source size of $10^{\prime \prime}$ (approximate size of the cluster of compact continuum sources seen in the SMA image of Hunter et al. 2006). Under this assumption, a least squares fit to the $\mathrm{H}^{35} \mathrm{Cl}$ spectrum gives an excitation temperature of $31 \mathrm{~K}$ and a column density of $4.0 \times 10^{14} \mathrm{~cm}^{-2}$. For $\mathrm{H}^{37} \mathrm{Cl}$, we derive an excitation temperature of $21 \mathrm{~K}$ and a column density of $1.5 \times 10^{14} \mathrm{~cm}^{-2}$; the resulting $\mathrm{H}^{35} \mathrm{Cl} / \mathrm{H}^{37} \mathrm{Cl}$ ratio is 2.7 . However, $\mathrm{HCl}$ column densities and the isotopic ratio depend strongly on the assumed source size (for a source size of $5^{\prime \prime}$ the derived isotopic ratio is 4.1 ). The $350 \mu \mathrm{m}$ continuum flux density toward NGC 6334I is 1430 Jy in a 9" beam (CSO/SHARC II; Dowell et al., private comm.) Assuming a dust temperature of $100 \mathrm{~K}$ (Sandell 2000) and a grain emissivity $\kappa_{350}=0.1 \mathrm{~cm}^{-2} \mathrm{~g}^{-1}$, we derive an $\mathrm{H}_{2}$ column density of $1.2 \times 10^{24} \mathrm{~cm}^{-2}$, which implies an $\mathrm{H}^{35} \mathrm{Cl}$ abundance of $\sim 1.7 \times 10^{-10}$ with respect to $\mathrm{H}$ nuclei. The lines of both $\mathrm{HCl}$ isotopologues are optically thick, with line center optical depths of $\sim 2.2$ and 1.6 for the strongest hyperfine component of $\mathrm{H}^{35} \mathrm{Cl}$ and $\mathrm{H}^{37} \mathrm{Cl}$, respectively.

\section{2. $S g r B 2(S)$}

The $\mathrm{p}-\mathrm{H}_{2}^{35} \mathrm{Cl}^{+}$spectrum towards Sgr B2(S) (Fig. 2, upper panel) shows strong absorption near the systemic velocity of the Sgr B2 envelope $\left(\sim 62 \mathrm{~km} \mathrm{~s}^{-1}\right)$ and two additional deep absorption components between 0 and $20 \mathrm{~km} \mathrm{~s}^{-1}$. In addition, shallow absorption is seen over a broad range of velocities down to $-100 \mathrm{~km} \mathrm{~s}^{-1}$, in agreement with the $\mathrm{H}$ I absorption spectrum towards the nearby source Sgr B2(M) (magenta line in Fig. 2). Both $\mathrm{H}^{35} \mathrm{Cl}$ and $\mathrm{H}^{37} \mathrm{Cl}$ (Fig. 2, lower panel) show deep absorption at the envelope velocity and a shallow absorption between 0

2 We made use of the myXCLASS program (http://www.astro. uni-koeln.de/projects/schilke/XCLASS), which accesses the CDMS (Müller et al. 2001; Müller et al. 2005; http://www . cdms . de) and JPL (Pickett et al. 1998; http: //spec . jpl . nasa.gov) molecular databases. 


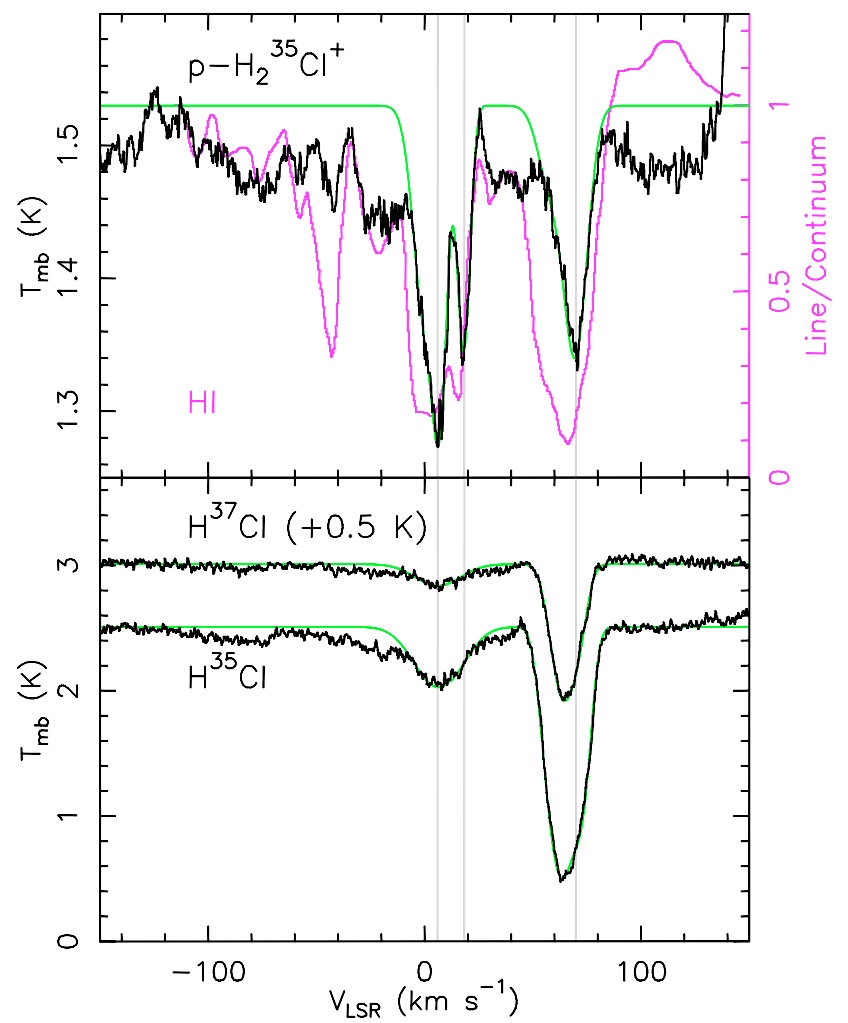

Fig. 2. Spectra of $\mathrm{p}-\mathrm{H}_{2}^{35} \mathrm{Cl}^{+} 1_{11}-0_{00}$ (upper panel), and $\mathrm{H}^{35} \mathrm{Cl}$ and $\mathrm{H}^{37} \mathrm{Cl}$ 1-0 (lower panel) towards $\mathrm{Sgr} \mathrm{B} 2(\mathrm{~S})$. The $\mathrm{H}^{37} \mathrm{Cl}$ spectrum has been shifted up by $0.5 \mathrm{~K}$. The magenta line in the upper panel shows the H I absorption spectrum towards Sgr B2(M) (from Garwood \& Dickey 1989).

and $20 \mathrm{~km} \mathrm{~s}^{-1}$. Similarly to NGC 6334I, we see velocity offsets of order a few $\mathrm{km} \mathrm{s}^{-1}$ between $\mathrm{HCl}$ and $\mathrm{H}_{2} \mathrm{Cl}^{+}$components.

Assuming a $5 \mathrm{~K}$ excitation temperature (the same as for NGC 6334I) and an ortho/para ratio of 3, we derive $\mathrm{H}_{2} \mathrm{Cl}^{+}$column densities of $3.4 \times 10^{13}$ and $2.2 \times 10^{13} \mathrm{~cm}^{-2}$ for the 0 and $62 \mathrm{~km} \mathrm{~s}^{-1}$ components, with corresponding $\mathrm{H}^{35} \mathrm{Cl}$ column densities of $4 \times 10^{13}$ and $2 \times 10^{14} \mathrm{~cm}^{2}$. The $\mathrm{H}^{35} \mathrm{Cl} / \mathrm{H}^{37} \mathrm{Cl}$ ratio is $\sim 3.3$ in both components. We estimate the uncertainties in our molecular column density estimates to be of order a factor of 2 .

To derive the hydrogen column density in the foreground gas towards Sgr B2(S), we use the method employed in Lis et al. (2001) to analyze the O I absorption towards Sgr B2(M), based on $\mathrm{H} \mathrm{I}$ and ${ }^{13} \mathrm{CO}$ absorption data. We assume that the foreground absorption is extended and column densities are the same towards Sgr B2(M) and (S). We derive a total hydrogen nuclei column density of $\sim 2 \times 10^{22} \mathrm{~cm}^{-2}$ in the atomic and molecular components in the velocity range -10 to $20 \mathrm{~km} \mathrm{~s}^{-1}$ (with a factor of 2 uncertainty). The corresponding chlorine content, in the form of $\mathrm{H}_{2} \mathrm{Cl}^{+}$and $\mathrm{HCl}$, is $7 \times 10^{13} \mathrm{~cm}^{-2}$, implying a $\mathrm{Cl} / \mathrm{H}$ ratio of $\sim 4 \times 10^{-9}$. This can be compared to the values measured in the UV in diffuse clouds (e.g., Sonnentrucker et al. 2006), which are in the range $3 \times 10^{-8}-4 \times 10^{-7}$. Therefore the high $\mathrm{H}_{2} \mathrm{Cl}^{+}$column densities we derive here are consistent with the overall chlorine budget, leaving plenty of room for atomic $\mathrm{Cl}$ and depletion on dust grains.

\section{Discussion}

Our estimates of the $\mathrm{H}_{2} \mathrm{Cl}^{+}$column densities towards NGC 6334I and Sgr B2(S), in excess of $10^{13} \mathrm{~cm}^{-2}$, are significantly higher than those expected for a single dense or diffuse PDR viewed at normal incidence. This might point to some deficiency in the models. Alternatively, a significant enhancement in the absorbing column density could result if the normal to the irradiated surface were inclined relative to the sight-line, or indeed if multiple PDRs were present along the sight-line, particularly if the radiation field is enhanced, as may be likely for the multiple absorption components seen towards Sgr B2. Similar discrepancies between models and observations are seen for other reactive ions in massive starforming regions (e.g., $\mathrm{CO}^{+}$ toward AFGL 2591; Bruderer et al. 2009).

We derive an $\mathrm{HCl} / \mathrm{H}_{2} \mathrm{Cl}^{+}$ratio of $\sim 10$ in $\mathrm{NGC} 6334 \mathrm{I}$ and the Sgr B2 envelope (assuming that in the case of NGC 6334I the $\mathrm{H}_{2} \mathrm{Cl}^{+}$column density on the back side is the same as that derived in front of the continuum source from our absorption measurements). This is well within the range predicted for dense PDRs (up to $\sim 100$ for densities above $10^{6} \mathrm{~cm}^{-3}$ ). The $\mathrm{HCl} / \mathrm{H}_{2} \mathrm{Cl}^{+}$ratio derived in the foreground gas towards Sgr B2(S) at velocities $0-20 \mathrm{~km} \mathrm{~s}^{-1}, \sim 1$, is also consistent with predictions of diffuse cloud models.

While a detailed analysis of chlorine chemistry in these and other sources that have been or will be observed using HIFI will be presented in a forthcoming paper, this work clearly demonstrates the outstanding spectroscopic capabilities of HIFI in the search for new interstellar molecules, particularly hydrides, and in providing robust constraints for astrochemical models of the interstellar medium.

\section{References}

Amin, M. Y. 1996, EMP, 73, 133

Araki, M., Furuya, T., \& Saito, S. 2001, J. Mol. Spec., 210, 132 Blake, G. A., Keene, J., \& Phillips, T. G. 1985, ApJ, 295, 501 Blake, G. A., Anacich, V. G., \& Huntress, W. T. 1986, ApJ, 300, 415 Brooks, K. J., \& Whiteoak, J. B. 2001, MNRAS, 320, 465 Bruderer, S., Benz, A. O., Doty, S. D., et al. 2009, ApJ, 700, 872 Burrus, Jr., C. A., Daniel, F., \& Gordy, W. 1953, Phys. Rev., 92, 274 Cernicharo, J., \& Guélin, M. 1987, A\&A, 183, L10 Cernicharo, J., Goicoechea, J. R., Daniel, F., et al. 2010, A\&A, 518, L115 Comito, C., \& Schilke, P. 2002, A\&A, 395, 357

Dalgarno, A., de Jong, T., Oppenheimer, M., \& Black., J. 1974, ApJ, 192, L37 de Graauw, Th., Helmich F. P., Phillips, T. G., et al. 2010, A\&A, 518, L6 de Leluw, F. H., \& Dymanus, A. 1973, J. Mol. Spec., 48, 427 Draine, B. T. 1978, ApJS, 36, 595

Emprechtinger, M., Lis, D. C., Bell, T., et al. 2010, A\&A, 521, L28

Endres, C. P., Drouin, B. J., Pearson, J. C., et al. 2009, A\&A, 504, 635

Federman, S. R., Cardell, Jason A., van Dishoeck, E. F., et al. 1995, ApJ, 445, 325

Garwood, R. W., \& Dickey, J. M. 1989, ApJ, 338, 841

Greaves, J. S., \& Nyman, L.-A. 1996, A\&A, 305, 950

Hunter, T. R., Brogan, C. L., Megeath, S. T., et al. 2006, ApJ, 649, 888

Jura, M. 1974, ApJ, 190, L33

Lis, D. C., Keene, J., Phillips, T. G., et al. 2001, ApJ, 561, 823

Müller, H. S. P., Thorwirth, S., Roth, D. A., \& Winnewisser, G. 2001, A\&A, 370, L49

Müller, H. S. P., Schlöder, F., Stutzki, J., \& Winnewisser, G. 2005, J. Mol. Struct., 742,215

Mürtz, P., Zink, L. R., Evenson, K. M., \& Brown, J. M. 1998, J. Chem. Phys., 109,9744

Neufeld, D. A., \& Wolfire, M. G. 2009, ApJ, 706, 1594 (NW09)

Ott, S. 2010, in Astronomical Data Analysis Software and Systems XIX, ed. Y. Mizumoto, K.-I. Morita, \& M. Ohishi, ASP Conf. Ser., in press

Pickett, H. M., Poynter, R. L., Cohen, E. A., et al. 1998, J. Quant. Spectrosc. Radiat. Transf., 60, 883

Pilbratt, G. L., Riedinger, J. R., Passvogel, T., et al. 2010, A\&A, 518, L1

Salez, M., Frerking, M. A., \& Langer, W. D. 1996, ApJ, 467, 708

Sandell, G. 2000, A\&A, 358, 242

Schilke, P., Phillips, T. G., \& Wang, N. 1995, ApJ, 441, 334

Schilke, P., Comito, C., Müller, H. S. P., et al. 2010, A\&A, 521, L11

Sonnentrucker, P., Friedman, S. D., \& York, D. G. 2006, ApJ, 650, L115

van der Wiel, M. H. D., van der Tak, F. F. S., Lis, D. C., et al. 2010, A\&A, 521, L43

van Dishoeck, E. F., \& Black, J. H. 1986, ApJS, 62, 109

Zmuidzinas, J., Blake, G. A., Carlstrom, J., et al. 1995, ApJ, 447, L125 
Table 1. Frequencies of the $\mathrm{H}_{2} \mathrm{Cl}^{+}$transitions observed.

\begin{tabular}{rcccc}
\hline \hline Transition & $\begin{array}{c}\text { Frequency } \\
(\mathrm{MHz})\end{array}$ & $\begin{array}{c}\text { Error } \\
(\mathrm{MHz})\end{array}$ & $\begin{array}{c}A_{\mathrm{ij}} \\
\left(\mathrm{s}^{-1}\right)\end{array}$ & $\begin{array}{c}E_{l} \\
\left(\mathrm{~cm}^{-1}\right)\end{array}$ \\
\hline \multicolumn{5}{c}{$\mathrm{H}_{2}^{35} \mathrm{Cl}^{+}$} \\
$1_{11}-0_{00} 3 / 2-3 / 2$ & 485413.427 & 0.029 & 0.00159 & 0 \\
$5 / 2-3 / 2$ & 485417.670 & 0.015 & 0.00159 & 0 \\
$1 / 2-3 / 2$ & 485420.796 & 0.057 & 0.00159 & 0 \\
$2_{12}-1_{01} 5 / 2-5 / 2$ & 781609.303 & 0.063 & 0.00179 & 14.1 \\
$3 / 2-1 / 2$ & 781611.062 & 0.062 & 0.00248 & 14.1 \\
$5 / 2-3 / 2$ & 781622.721 & 0.063 & 0.00417 & 14.1 \\
$7 / 2-5 / 2$ & 781626.794 & 0.060 & 0.00596 & 14.1 \\
$1 / 2-1 / 2$ & 781628.554 & 0.061 & 0.00496 & 14.1 \\
$3 / 2-3 / 2$ & 781635.214 & 0.062 & 0.00318 & 14.1 \\
\hline \multicolumn{5}{c}{$\mathrm{H}_{2}^{37} \mathrm{Cl}^{+}$} \\
& & & \\
$2_{12}-1_{01} 5 / 2-5 / 2$ & 780037.315 & 0.069 & 0.00178 & 14.1 \\
$3 / 2-1 / 2$ & 780038.760 & 0.066 & 0.00247 & 14.1 \\
$5 / 2-3 / 2$ & 780047.903 & 0.066 & 0.00414 & 14.1 \\
$7 / 2-5 / 2$ & 780051.197 & 0.062 & 0.00592 & 14.1 \\
$1 / 2-1 / 2$ & 780052.642 & 0.065 & 0.00493 & 14.1 \\
$3 / 2-3 / 2$ & 780057.820 & 0.068 & 0.00316 & 14.1 \\
\hline
\end{tabular}

Notes. Frequencies and spontaneous emission coefficients have been calculated from the constants derived by Araki et al. (2001), see also CDMS.

Acknowledgements. HIFI has been designed and built by a consortium of institutes and university departments from across Europe, Canada and the United States under the leadership of SRON Netherlands Institute for Space Research, Groningen, The Netherlands and with major contributions from Germany, France and the US. Consortium members are: Canada: CSA, U. Waterloo; France: CESR, LAB, LERMA, IRAM; Germany: KOSMA, MPIfR, MPS; Ireland, NUI Maynooth; Italy: ASI, IFSI-INAF, Osservatorio Astrofisico di Arcetri- INAF; Netherlands: SRON, TUD; Poland: CAMK, CBK; Spain: Observatorio Astronomico Nacional (IGN), Centro de Astrobiología (CSIC-INTA). Sweden: Chalmers University of Technology - MC2, RSS \& GARD; Onsala Space Observatory; Swedish National Space Board, Stockholm University - Stockholm Observatory; Switzerland: ETH Zurich, FHNW; USA: Caltech, JPL, NHSC. Support for this work was provided by NASA through an award issued by JPL/Caltech. D. C. L. is supported by the NSF, award AST-0540882 to the CSO. A portion of this research was performed at the Jet Propulsion Laboratory, California Institute of Technology, under contract with the National Aeronautics and Space Administration.

1 California Institute of Technology, Cahill Center for Astronomy and Astrophysics 301-17, Pasadena, CA 91125, USA

e-mail: dcl@caltech.edu

2 Department of Astronomy, University of Michigan, 500 Church Street, Ann Arbor, MI 48109, USA

3 Department of Physics and Astronomy, Johns Hopkins University, 3400 North Charles Street, Baltimore, MD 21218, USA

${ }^{4}$ Centre d'Étude Spatiale des Rayonnements, Université de Toulouse [UPS], 31062 Toulouse Cedex 9, France

5 CNRS/INSU, UMR 5187, 9 avenue du Colonel Roche, 31028 Toulouse Cedex 4, France

${ }^{6}$ Laboratoire d'Astrophysique de l'Observatoire de Grenoble, BP 53, 38041 Grenoble Cedex 9, France

7 Centro de Astrobiología (CSIC/INTA), Laboratiorio de Astrofísica Molecular, Ctra. de Torrejón a Ajalvir, km 4, 28850 Torrejón de Ardoz, Madrid, Spain
8 Max-Planck-Institut für Radioastronomie, Auf dem Hügel 69, 53121 Bonn, Germany

9 LERMA, CNRS UMR8112, Observatoire de Paris and École Normale Supérieure, 24 rue Lhomond, 75231 Paris Cedex 05, France

10 LPMAA, UMR7092, Université Pierre et Marie Curie, Paris, France

11 LUTH, UMR8102, Observatoire de Paris, Meudon, France

12 I. Physikalisches Institut, Universität zu Köln, Zülpicher Str. 77, 50937 Köln, Germany

13 Jet Propulsion Laboratory, Caltech, Pasadena, CA 91109, USA

14 Departments of Physics, Astronomy and Chemistry, Ohio State University, Columbus, OH 43210, USA

15 National Research Council Canada, Herzberg Institute of Astrophysics, 5071 West Saanich Road, Victoria, BC V9E 2E7, Canada

16 Infrared Processing and Analysis Center, California Institute of Technology, MS 100-22, Pasadena, CA 91125, USA

17 Canadian Institute for Theoretical Astrophysics, University of Toronto, 60 St George St, Toronto, ON M5S 3H8, Canada

18 Harvard-Smithsonian Center for Astrophysics, 60 Garden Street, Cambridge MA 02138, USA

19 National University of Ireland, Maynooth, Ireland

20 SRON Netherlands Institute for Space Research, PO Box 800, 9700 AV, Groningen, The Netherlands

21 Department of Physics and Astronomy, University of Calgary, 2500 University Drive NW, Calgary, AB T2N 1N4, Canada

22 Department of Astronomy, University of Massachusetts, Amherst, MA, USA

23 Université de Bordeaux, Laboratoire d'Astrophysique de Bordeaux, France; CNRS/INSU, UMR 5804, Floirac, France

24 INAF - Istituto di Fisica dello Spazio Interplanetario, Roma, Italy

25 Observatoire de Paris, LERMA UMR CNRS 8112, France

26 School of Physics and Astronomy, University of Leeds, Leeds UK

27 INAF Osservatorio Astrofisico di Arcetri, Florence, Italy

28 Astronomical Institute "Anton Pannekoek", University of Amsterdam, Amsterdam, The Netherlands

29 Department of Astrophysics/IMAPP, Radboud University Nijmegen, Nijmegen, The Netherlands

30 IGN Observatorio Astronómico Nacional, Alcalá de Henares, Spain

31 INAF - Osservatorio Astronomico di Roma, Monte Porzio Catone, Italy

32 INAF - Istituto di Fisica dello Spazio Interplanetario, Roma, Italy

33 Institut de RadioAstronomie Millimétrique, Grenoble, France

${ }^{34}$ Leiden Observatory, Leiden University, Leiden, The Netherlands

35 Department of Physics and Astronomy, University College London, London, UK

36 Department of Radio \& Space Science, Chalmers University of Technology, Onsala, Sweden

37 Institute of Astronomy, ETH-Zurich, Zurich, Switzerland

38 Onsala Space Observatory, Chalmers Institute of Technology, Onsala, Sweden

39 SETI Institute, Mountain View, CA, USA

40 Department of Physics and Astronomy, San Jose State University, San Jose, CA, USA

41 Department of Astronomy, Stockholm University, Stockholm, Sweden

42 Observatoire de Paris, LUTH, and Université Denis Diderot, Meudon, France

43 Stockholm Observatory, Stockholm, Sweden

44 Institute Laboratoire d'Études du Rayonnement et de la Matière en Astrophysique, UMR 8112 CNRS/INSU, OP, ENS, UPMC, UCP, Paris, France and LERMA, Observatoire de Paris, Paris, France

45 Max-Planck-Institut für Astronomie, Heidelberg, Germany

46 Kapteyn Astronomical Institute, University of Groningen, The Netherlands 